\title{
Editorial
}

\section{Geoinformatics in Citizen Science}

\author{
Gloria Bordogna \\ CNR_IREA, via A. Corti 12, 20133 Milano, Italy; bordogna.g@irea.cnr.it; Tel.: +39-02-23699-299
}

Received: 29 November 2018; Accepted: 6 December 2018; Published: 11 December 2018

\begin{abstract}
This editorial introduces the special issue entitled "Geoinformatics in Citizen Science" of the ISPRS International Journal of Geo-Information. The issue includes papers dealing with three main topics. (1) Key tasks of citizen science (CS) in leveraging geoinformatics. This comprises descriptions of citizen science initiatives where geoinformation management and processing is the key means for discovering new knowledge, and it includes: (i) "hackAIR: Towards Raising Awareness about Air Quality in Europe by Developing a Collective Online Platform" by Kosmidis et al., (ii) "Coupling Traditional Monitoring and Citizen Science to Disentangle the Invasion of Halyomorpha halys" by Malek et al., and (iii) "Increasing the Accuracy of Crowdsourced Information on Land Cover via a Voting Procedure Weighted by Information Inferred from the Contributed Data" by Foody et al. (2) Evaluations of approaches to handle geoinformation in CS. This examines citizen science initiatives which critically analyze approaches to acquire and handle geoinformation, and it includes: (iv) "CS Projects Involving Geoinformatics: A Survey of Implementation Approaches" by Criscuolo et al., (v) "Obstacles and Opportunities of Using a Mobile App for Marine Mammal Research" by Hann et al., (vi) "OSM Data Import as an Outreach Tool to Trigger Community Growth? A Case Study in Miami" by Juhász and Hochmair, and (vii) "Experiences with Citizen-Sourced VGI in Challenging Circumstances" by Hameed et al. (3) Novel geoinformatics research issues: (viii) "A New Method for the Assessment of Spatial Accuracy and Completeness of OpenStreetMap Building Footprints" by Brovelli and Zamboni, (ix) "A Citizen Science Approach for Collecting Toponyms" by Perdana and Ostermann, and (x) "An Automatic User Grouping Model for a Group Recommender System in Location-Based Social Networks" by Khazaei and Alimohammadi.
\end{abstract}

Keywords: geoinformation in citizen science; VGI in citizen science; crowdsourced geoinformation collection and analysis

\section{Introduction}

The idea of editing this special issue was motivated by the observation of the increasing number of academic papers focused on the characteristics of volunteered geographic information (VGI) and crowdsourced geoinformation within citizen science (CS) projects, and on evaluations of the potential for VGI to help scientists, policy makers, and business companies in conceiving and launching new scientific projects [1-8]. VGI and crowdsourced geoinformation from social networks are being investigated as a novel opportunity to launch research projects with widespread ground data, including monitoring of natural, environmental, human-driven, and social changes and events. In these contexts, VGI appears as a relevant aspect of CS. Nevertheless, collecting VGI, filtering crowdsourced geoinformation from its sources, and analyzing it implies the adoption and application of geoinformatics techniques which were first developed for managing traditional geodata in GIS environments. Thus, the appropriateness, coverage, adaptability, and completeness of traditional geoinformation technologies to manage VGI and crowdsourced information in CS deserve an investigation. 
The vast literature describing CS initiatives do not specifically focus on the geoinformatics algorithms and technologies applied in relation to the activities and tasks of the projects. This may be due to the fact that the community of researchers in CS is generally very heterogeneous, spanning from experts in various CS application domains, to social scientists studying crowd participation and volunteers' characteristics, and finally, to computer scientists who are often involved in CS activities as mere executors and implementers of solutions. The objectives of this special issue were to overview the latest geoinformation processing approaches used in CS initiatives to investigate CS activities and tasks that can benefit from the analysis of geoinformation, to envisage ongoing technological solutions and trends for geoinformatics in CS, and finally, to outline problems and unsolved issues.

This special issue received a total of 13 submitted papers with 10 papers accepted [9-18].

The authors' affiliations are distributed in the following countries: Austria, Germany, Greece, Italy, Netherlands, Norway, the United Kingdom, and the United States. The described CS initiatives span several geographic areas: Indonesia, Germany, Norway, Italy, the United States, and Iraq.

Topics covered include three main parts: (1) CS key tasks in leveraging geoinformatics, (2) evaluations of approaches to handle geoinformation in CS, and (3) novel geoinformatics research issues. The three topics and accepted papers are briefly described below.

\section{CS Key Tasks in Leveraging Geoinformatics}

Within this section, we examine descriptions of CS initiatives where geoinformation management and processing are the key means needed for pursuing the objectives of the CS projects (i.e., for discovering new knowledge on the specific application domain of the projects, or for performing some relevant activity of the project, such as reliable geodata filtering, management, analysis, synthesis, sharing, and visualization. This topic includes the following papers: (i) "hackAIR: Towards Raising Awareness about Air Quality in Europe by Developing a Collective Online Platform" by Kosmidis et al., (ii) "Coupling Traditional Monitoring and Citizen Science to Disentangle the Invasion of Halyomorpha halys" by Malek et al., and (iii) "Increasing the Accuracy of Crowdsourced Information on Land Cover via a Voting Procedure Weighted by Information Inferred from the Contributed Data" by Foody et al.

(i) "hackAIR: Towards Raising Awareness about Air Quality in Europe by Developing a Collective Online Platform" by Kosmidis et al. well exemplifies some geoinformatics techniques which can be useful for crowdsourced multimedia data filtering and geolocating, multisource geoinformation merging in order to provide improved and more complete information in areas with partial and missing measurements, and personalized recommendations to citizens based on their profiles and areas. Motivated by the observation that air quality data are often scarce, the paper proposed a centralized air quality data hub with a loosely coupled service-oriented architecture. They applied up-to-date methods to collect multisource information from low-cost sensors and official measurement stations and consolidated technologies to merge these data with crowdsourced information filtered from social media (i.e., geotagged sky-depicting photos from Flicker and official webcam images). To automatically detect the presence of sky in an image, a visual concept detection model using deep convolutional neural networks was applied. Then, the location of the depicted sky was identified by applying a rule-based approach which was evaluated as yielding greatest performance with respect to using a fully convolutional network. Citizens can contribute to air quality monitoring by building and using low-cost sensing devices that optically determine air particles by means of a light scattering method. Finally, a data fusion algorithm based on geostatistics (i.e., universal kriging for interpolating the observations in space using model information as a spatial proxy) interpolated the point-based observations in space such that air quality estimates were available at any point within the domain. Since the final aim of the project was to provide personalized tips on how citizens can reduce their ecological footprint or personalized advice on how individuals may respond to existing atmospheric conditions, ontologies and semantic web technology were used for structuring and semantically integrating data. 
(ii) "Coupling Traditional Monitoring and Citizen Science to Disentangle the Invasion of Halyomorpha halys" by Malek et al. In describing the "BugMap" science initiative to complement traditional ecological surveys and assist researchers in breaking down the behavior of invasive pests via a user-friendly and freely available mobile application, this paper well illustrates how social media, mobile platforms, and GIS can aid in recruiting and training volunteers to create observations and building species distribution models. The models were built by locality data geocorrelation with environmental variables extracted from authoritative geodata using GIS technologies. Specifically, the MaxEnt software package, a machine learning algorithm that applies the principle of maximum entropy, was used to predict the probability of the spatial distribution of species from presence-only data, represented by a Gaussian kernel function and environmental variables. Sensitivity analysis was performed by varying parameters and computing the receiver operating characteristic (ROC) curve to compare the area under the ROC curve (AUC) of all the models in order to identify the best bias treatment solution for the case study. The paper also reports an interesting geotemporal analysis of the characteristics of both the locations where volunteers created their observations and the species distribution.

(iii) "Increasing the Accuracy of Crowdsourced Information on Land Cover via a Voting Procedure Weighted by Information Inferred from the Contributed Data" by Foody et al. faces the critical issue of filtering reliable VGI to determine an ensemble classification of contributions which could be considered as the agreed classification of the crowd regarded as a unique contributing entity. In this work, the wisdom of the crowd was extrapolated by applying consensus dynamic models taking into account the geolocation of volunteers and their contributions; specifically, the paper explored how to increase the accuracy of crowdsourced data on land cover identified from satellite remote sensing images through the use of weighted voting strategies. Different consensus strategies were tested: the simple majority voting approach and several weighted voting strategies, in which both contributors' skills and models' parameters were considered. The results show that consensus approaches can aid in filtering reliable crowdsourced data and contributors with high agreement, so as to yield an ensemble classification that is more accurate than that achieved by any individual contributor.

\section{Evaluation of Approaches to Handle Geoinformation in CS}

This section includes CS initiatives, the focus of which is to analyze and critically evaluate approaches to create and manage geoinformatics that can be adopted for a given task in CS. It includes: (iv) "CS Projects Involving Geoinformatics: A Survey of Implementation Approaches" by Criscuolo et al., (v) "Obstacles and Opportunities of Using a Mobile App for Marine Mammal Research" by Hann et al., (vi) "OSM Data Import as an Outreach Tool to Trigger Community Growth? A Case Study in Miami" by Juhász and Hochmair, and (vii) "Experiences with Citizen-Sourced VGI in Challenging Circumstances" by Hameed et al.

(iv) "CS Projects Involving Geoinformatics: A Survey of Implementation Approaches" by Criscuolo et al. As stated in the title, this work tackled the objective of analyzing diversified ongoing CS initiatives from the perspective of geoinformation approaches they adopted for the various tasks of a CS project in action. To this end, they first proposed a common conceptualization of the CS activity workflow, from data generation and delivery, data visualization and access, data processing, to data qualification and validation. Then, a multidimensional classification of the selected CS initiatives was proposed in which each dimension, corresponding to a phase of the CS workflow, was categorized with respect to several main implementation approaches that can be applied. The final aim is to understand which are the most common and used approaches of geoinformatics actually employed in CS and how they evolved.

(v) "Obstacles and Opportunities of Using a Mobile App for Marine Mammal Research" by Hann et al. tackles the up-to-date issue of critically investigating how the use of a mobile application called Whale mAPP (www.whalemapp.org) for recording georeferenced opportunistic 
marine mammal sighting data in southeast Alaska impacts both the recruitment and commitment of contributors and the quality of VGI. Besides these objectives, the paper also included evaluating the potential educational and scientific benefits and limitations of mobile application use for the purpose of improving future CS projects. To achieve the educational objectives, citizen scientists completed a questionnaire before and after using the mobile app to assess participants' motivations, general experience, and educational outcomes of using the app. Technological glitches and participant retention added additional insight.

(vi) "OSM Data Import as an Outreach Tool to Trigger Community Growth? A Case Study in Miami" by Juhász and Hochmair presents the results of a study that explored if and how an OpenStreetMap (OSM) data import tool can contribute to OSM community growth. The software tool implements a hybrid approach for the building import task that consists of an automated bulk upload of buildings and a manual community review of the remaining buildings. A custom workflow using JOSM editor was developed and explained in a detailed tutorial to three targeted OSM user groups, namely, existing OSM members, local mappers, and students recruited to this purpose. The paper analyzed the spatiotemporal user contributions of the target groups of volunteers. Results revealed differences in editing patterns between newly recruited users and already-established mappers. More specifically, long-term engagement of newly registered OSM mappers did not succeed, whereas already-established contributors continued to import and improve data. In general, they found that an OSM data import tool can add valuable data to the map, but also that encouraging long-term engagement of new users, within or outside the academic environment, proves to be challenging.

(vii) "Experiences with Citizen-Sourced VGI in Challenging Circumstances" by Hameed et al. explores the process of VGI collection by assessing the relative usability and accuracy of a range of different means and methods for data collection among different demographic and educational groups and in different geographical contexts within a study area: smartphone with a GPS app installed for locating land parcel corners and attributing the resultant polygon; portable iPad Tablet PC with the official cadastral map uploaded and overwriting and annotating capability provided through the open source QGIS; and finally, paper-printed aerial or satellite images, with clipboard and pencil for demarcation and annotation. Assessments were made of positional accuracy, completeness, and the experiences of citizen data collectors with reference to the official cadastral data and the land administration system. Ownership data were validated by crowd agreement. The outcomes of this research show the varying effects of volunteers in relation with data collection method, geographical area, and application field.

\section{Novel Geoinformatics Research Issues}

This section groups three articles that exhibit novelty with respect to the geoinformatics approach they apply, analyze, or propose to perform regarding a specific task in a CS initiative. It includes: (viii) "A New Method for the Assessment of Spatial Accuracy and Completeness of OpenStreetMap Building Footprints" by Brovelli and Zamboni (ix) "A Citizen Science Approach for Collecting Toponyms" by Perdana and Ostermann, and (x) "An Automatic User Grouping Model for a Group Recommender System in Location-Based Social Networks" by Khazaei and Alimohammadi.

(viii) "A New Method for the Assessment of Spatial Accuracy and Completeness of OpenStreetMap Building Footprints" by Brovelli and Zamboni. Although tackling the very common topic of spatial accuracy evaluation of OSM data, it proposes an original artificial intelligence geoinformatics approach which mimics human behavior when making comparisons of maps. Specifically, the assessment of the spatial accuracy is based on the evaluation of the distance between points representing the same features in two different maps (or layers) depicting the same area. The implemented algorithm works on vector layers considering the vertices of the map featured as a set of coordinates. In detecting the homologous entity (the study case, the building footprint), it compares the position, shape, and semantics of the features on the two 
maps like a human being would. Finding such a correspondence is not trivial, since the two maps could both have slightly different scales and not exactly the same level of details. The comparison must then cope with vagueness and imprecision.

(ix) "A Citizen Science Approach for Collecting Toponyms" by Perdana and Ostermann. This research article starts from the assessment that crowdsourced geographic information and citizen science approaches can offer a new paradigm of toponym collection and addresses issues in advancing toponym practices. It starts by systematically examining the current state of the art of toponym collection and handling practices by multiple stakeholders and then identifies a recurring set of problems. Furthermore, it develops a citizen science approach, based on a crowdsourcing level of participation, to collect toponyms. The proposal identifies the minimum requirements that future mobile and web applications should have for collecting toponyms; specifically, nine essential functionalities are deemed important: navigation, marking GPS coordinates, tracking, displaying a map, taking geo-tagged photos, recording audio, ability to create geo-tagged notes or the generation of forms, offline functionality, and user friendliness and simple user interface. Finally, the implementation of the proposal in the context of an Indonesian case study is discussed.

(x) "An Automatic User Grouping Model for a Group Recommender System in Location-Based Social Networks" by Khazaei and Alimohammadi considers the problem of spatial group recommendations for suggesting places to a given set of users. In a group recommender system, members of a group should have similar preferences in order to increase the level of satisfaction. In this paper, an automatic user grouping model is introduced that obtains information about the preferences of the users, proximity of the places the users have visited in terms of spatial range, users' free days, and the social relationships among users automatically from location histories and users' profiles. These factors are combined to determine the similarities among users. The users are partitioned into groups based on these similarities. Notice that CS could leverage spatial group recommendation for several purposes, for example, for making suggestions of new areas to visit to contributors based on areas visited by others with similar preferences, so as to encourage user long-term commitment, which was identified as one major weak point of CS initiatives.

\section{Conclusions}

When I undertook the editing of this special issue, I expected to receive many contributions relative to VGI and sensor data interoperable web sharing, semantic representation and management of volunteers' contributions, and credibility/reliability/accuracy assessments of both volunteers and their contributions. Only the last topic is covered by the received papers, probably hinting at the fact that interoperability and semantic issues are solved problem. Many of the papers investigate or discuss the use of mobile applications as a suitable means for both collecting high-quality contributions and engaging long-term contributors. This testifies to the fact that mobile technologies are pervading our life habits, and thus, CS initiatives are investigating if and how mobile applications can constitute a potential to empower CS.

Some unexpected topics were also covered by the papers, such as the use of both machine learning algorithms and artificial intelligence, probably on the wave of popularity of these approaches.

I want to express my congratulation to the authors of the papers for their interesting works; my gratitude to the anonymous referees, whose excellent work made it possible to improve the contents of the papers; and finally, my thanks to the editorial staff of the IJGI for the assistance in producing this special issue.

Funding: This work was supported by URBAN-GEO BIG DATA, a Project of National Interest (PRIN) funded by the Italian Ministry of Education, University and Research (MIUR)—ID. 20159CNLW8.

Conflicts of Interest: The author declares no conflict of interest. 


\section{References}

1. Goodchild, M.; Aubrecht, C.; Bhaduri, B. Special Issue Role of Volunteered Geographic Information in Advancing Science. Trans. GIS 2016. Available online: http:/ / onlinelibrary.wiley.com/doi/10.1111/tgis. 12242/full (accessed on 4 July 2017).

2. Bordogna, G.; Carrara, P. (Eds.) Mobile Information Systems Leveraging Volunteered Geographic Information for Earth Observation; Earth Systems Data and Models Series; Springer: Heidelberg, Germany, 2018.

3. Mooney, P.; Zipf, A.; Jokar, J.; Hochmair, H.H. AGILE Workshop on VGI-Analytics. 2017. Available online: http:/ / www.cs.nuim.ie/ \{\}pmooney/VGI-Analytics2017/ (accessed on 4 July 2017).

4. Mooney, P.; Zipf, A.; Jokar, J.; Hochmair, H.H. Special issue on Volunteered Geographic Information (VGI)-Analytics. Forthcoming in Geo-spatial Information Science, late 2017. 2017. Available online: http: / / explore.tandfonline.com/cfp/est/gsis/si3 (accessed on 4 July 2017).

5. Pfoser, D.; Voisard, A. GEOCROWD Workshop Report: The Second Int'l Workshop on Crowdsourced and Volunteered Geographic Information 2013: (Orlando, FL-Nov. 5, 2013); SIGSPATIAL Special; ACM: New York, NY, USA, 2014; Volume 6, p. 11.

6. See, L.; Fritz, S.; de Leeuw, J. Special Issue Collaborative Mapping. ISPRS Int. J. Geo-Inf. 2013, 2, $955-958$. [CrossRef]

7. Zipf, A.; Jonietz, D.; Antoniou, V.; See, L. Special Issue Volunteered Geographic Information. ISPRS Int. J. Geo-Inf. 2017. Available online: http://www.mdpi.com/journal/ijgi/special_issues/VGI (accessed on 4 July 2017).

8. Zipf, A.; Resch, B. Special Issue on GeoWeb 2.0. ISPRS Int. J. Geo-Inf. 2015. Available online: http: / / www.mdpi.com/journal/ijgi/special_issues/geoweb-2.0 (accessed on 4 July 2017).

9. Criscuolo, L.; Bordogna, G.; Carrara, P.; Pepe, M. CS Projects Involving Geoinformatics: A Survey of Implementation Approaches. ISPRS Int. J. Geo-Inf. 2018, 7, 312. [CrossRef]

10. Brovelli, M.A.; Zamboni, G. A New Method for the Assessment of Spatial Accuracy and Completeness of OpenStreetMap Building Footprints. ISPRS Int. J. Geo-Inf. 2018, 7, 289. [CrossRef]

11. Perdana, A.P.; Ostermann, F.O. A Citizen Science Approach for Collecting Toponyms. ISPRS Int. J. Geo-Inf. 2018, 7, 222. [CrossRef]

12. Kosmidis, E.; Syropoulou, P.; Tekes, S.; Schneider, P.; Spyromitros-Xioufis, E.; Riga, M.; Charitidis, P.; Moumtzidou, A.; Papadopoulos, S.; Vrochidis, S.; et al. hackAIR: Towards Raising Awareness about Air Quality in Europe by Developing a Collective Online Platform. ISPRS Int. J. Geo-Inf. 2018, 7, 187. [CrossRef]

13. Malek, R.; Tattoni, C.; Ciolli, M.; Corradini, S.; Andreis, D.; Ibrahim, A.; Mazzoni, V.; Eriksson, A.; Anfora, G. Coupling Traditional Monitoring and Citizen Science to Disentangle the Invasion of Halyomorpha halys. ISPRS Int. J. Geo-Inf. 2018, 7, 171. [CrossRef]

14. Hann, C.H.; Stelle, L.L.; Szabo, A.; Torres, L.G. Obstacles and Opportunities of Using a Mobile App for Marine Mammal Research. ISPRS Int. J. Geo-Inf. 2018, 7, 169. [CrossRef]

15. Juhász, L.; Hochmair, H.H. OSM Data Import as an Outreach Tool to Trigger Community Growth? A Case Study in Miami. ISPRS Int. J. Geo-Inf. 2018, 7, 113. [CrossRef]

16. Foody, G.; See, L.; Fritz, S.; Moorthy, I.; Perger, C.; Schill, C.; Boyd, D. Increasing the Accuracy of Crowdsourced Information on Land Cover via a Voting Procedure Weighted by Information Inferred from the Contributed Data. ISPRS Int. J. Geo-Inf. 2018, 7, 80. [CrossRef]

17. Khazaei, E.; Alimohammadi, A. An Automatic User Grouping Model for a Group Recommender System in Location-Based Social Networks. ISPRS Int. J. Geo-Inf. 2018, 7, 67. [CrossRef]

18. Hameed, M.; Fairbairn, D.; Speak, S. Experiences with Citizen-Sourced VGI in Challenging Circumstances. ISPRS Int. J. Geo-Inf. 2017, 6, 385. [CrossRef]

(C) 2018 by the author. Licensee MDPI, Basel, Switzerland. This article is an open access article distributed under the terms and conditions of the Creative Commons Attribution (CC BY) license (http://creativecommons.org/licenses/by/4.0/). 